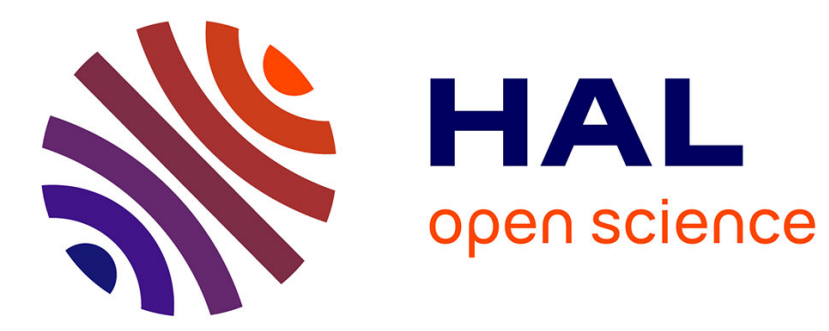

\title{
Efficient Deployment of Connected Unmanned Aerial Vehicles for Optimal Target Coverage
}

\author{
Christelle Caillouet, Tahiry Razafindralambo
}

\section{To cite this version:}

Christelle Caillouet, Tahiry Razafindralambo. Efficient Deployment of Connected Unmanned Aerial Vehicles for Optimal Target Coverage. GIIS 2017 - Global Information Infrastructure and Networking Symposium, Oct 2017, Saint-Pierre, France. 10.1109/GIIS.2017.8169803 . hal-01575618

\section{HAL Id: hal-01575618 \\ https://hal.inria.fr/hal-01575618}

Submitted on 21 Aug 2017

HAL is a multi-disciplinary open access archive for the deposit and dissemination of scientific research documents, whether they are published or not. The documents may come from teaching and research institutions in France or abroad, or from public or private research centers.
L'archive ouverte pluridisciplinaire HAL, est destinée au dépôt et à la diffusion de documents scientifiques de niveau recherche, publiés ou non, émanant des établissements d'enseignement et de recherche français ou étrangers, des laboratoires publics ou privés. 


\section{Efficient Deployment of Connected Unmanned Aerial Vehicles for Optimal Target Coverage}

\author{
Christelle Caillouet \\ Université Côte d'Azur, CNRS, Inria, I3S, France
}

\author{
Tahiry Razafindralambo \\ Université La Réunion, LIM, France
}

\begin{abstract}
Anytime and anywhere network access can be provided by Unmanned Aerial Vehicles (UAV) with air-to-ground and air-to-air communications using directional antennas for targets located on the ground. Deploying these Unmanned Aerial Vehicles to cover targets is a complex problem since each target should be covered, while minimizing (i) the deployment cost and (ii) the UAV altitudes to ensure good communication quality. We also consider connectivity between the UAVs and a base station in order to collect and send information to the targets, which is not considered in many similar studies. In this paper, we provide an efficient optimal program to solve this problem and show the trade-off analysis due to conflicting objectives. We propose a fair trade-off optimal solution and also evaluate the cost of adding connectivity to the UAV deployment.
\end{abstract}

\section{INTRODUCTION}

Providing network services access anytime and anywhere has become an important challenge during the recent years. This challenge is worsen by the environmental context such as vehicular networks, environmental monitoring in harsh conditions or disaster recovery. For the later, providing ground access to network services is almost impossible. However, fair quality of emergency services must be provided. A natural response to such a need is the use of autonomous flying devices or Unmanned Aerial Vehicles (UAV) to provide the needed services to targets on the ground. UAVs with air-to-ground and air-to-air communications using directional antennas provide potential solution to anytime and anywhere network access.

Deploying Unmanned Aerial Vehicles to cover targets is a complex problem. Indeed, the deployment should minimize the number of UAVs to reduce the cost. UAVs should be optimally placed to cover all the targets while minimizing overlapped coverage UAVs. We assume in the latter that coverage is related to directional antenna pointing to the ground for communication. Moreover, high quality wireless air-to-ground communication should be provided between the UAVs and the ground targets by minimizing the altitude of the UAVs. Depending on the area size to be covered by the UAVs, multi-hop connectivity should be provided between UAVs and a base station using air-to-air communications. Full coverage of the targets, coverage quality and connectivity are important objectives and constraints of our problem.

The use of flying devices to cover ground targets has become an important topic for the past few years. Research studies concern both theoretical and practical considerations of the topic. From practical point of view, some researches focus on distributed deployment of UAVs and from theoretical point of view, researches focus on optimal deployment. To the best of our knowledge, neither from practical point of view, nor from theoretical point of view, a problem tackling the optimal full coverage optimizing the coverage quality and connectivity in a 3D environment has been addressed. Combining these constraints and objectives is very important since it can help reducing the cost of UAV deployment and increase their usage.

Many research focus on UAVs or robots deployment in the literature. For example in [1], authors considered the same type of problem as the one we want to tackle. However their assumptions are different. Authors do not ensure connectivity among the UAVs, and their primary focus is energy consumption. We have seen that ensuring UAV connectivity with a fixed base station is interesting to collect efficiently information from the ground to a central entity in order to be efficiently analyzed. In [2], authors consider maximizing the total coverage area of the UAVs and their lifetime. But in their model, all UAVs are assumed to be placed at the same altitude and thus do not consider possible coverage quality. Moreover, the authors of [2] do not ensure connectivity between each UAV. In both [1] and [2] the main and most important difference with our work is the UAV connectivity. We think that this constraint is very important since it ensures uninterrupted bi-directional information exchanges between the base station and the targets.

In this paper, we consider the following problem : Given a set of targets deployed on the ground, the goal is to cover all the targets at minimum cost with drones flying as low as possible. Moreover, we ensure that the UAVs form a connected graph with a fixed base station in order to collect and analyze efficiently the information. We show that it is possible to develop an efficient model that computes optimal positions of the UAVs. We also show that coverage problem (minimizing the deployment cost and the UAVs altitude) and connectivity should be optimized using Pareto optimality concept. And finally we show that the connectivity constraint have a non negligible effect on the number of deployed UAVs.

The remainder of this paper is organized as follows. In Section II, we review the state of the art. Section III provides some background, describes the deployment problem and gives a linear model and its two objective functions. Results are given in IV. They describe the efficiency of our model by analyzing the trade-off between deployment cost and altitude, and give an evaluation of the connectivity cost. Conclusions are drawn in Section V. 


\section{RELATED WORK}

Full target coverage by a fleet of UAVs or mobile devices have been studied by many different viewpoints but especially from two research communities. From the robotics point of view, the main objective is the collaboration among mobile devices for a specific task. The robotic community focuses on the deployment itself or how to drive each device to its specific location. From the ad hoc and sensor network community, given a location for each device, the objective is to implement protocols above the deployed network. Another way to tackle the issue is from the optimization point of view. A lot of work have been focusing on optimally choosing the location of each mobile device such as in [3], [4], [5], [6]. In these works related to UAV location problem, the authors assume that the devices evolve in a $2 \mathrm{D}$ space. Therefore their problem is simplified because the coverage radius is fixed for each mobile devices. It is worth noting that some solutions provided in the cited works can be modified to take into account different coverage radius and thus simulating UAV altitude.

In [7], Kar et al. provide a study on node placement including connectivity constraints. However, the provided solution is not optimal and is based on approximation. Moreover, the algorithms are designed for 2D space in which coverage radius is fixed. In [8] the authors focus on linear connected topology construction. In contrast, we focus on a 3D model. An interesting point raised by the authors of [8] is related to the coverage aspect. They assume that coverage is an attenuated disc. In our case, we assume a complete and full coverage of each specific target.

The closest works to ours are from Zorbas et al. in [1] and [9] and Pugliese et al. in [10]. In [10] the authors provide a reference model to compare the target covering problem using flying drones using a mixed integer programming formulation. The solution proposed in [10] is then used in [1] as a reference model to their distributed solution. The problem both papers try to solve is related to energy consumption. Two metrics are considered for the cost; the number of UAVs and the total energy consumption. It is assumed that each UAV has a minimum and a maximum altitude and the energy consumption is related to its altitude. In their model, the authors of [9] and [10] use the same assumption as we do in this paper. However, the fundamental difference between their works and ours lays in the constraints. Indeed, we consider connectivity as a fundamental characteristic of a network and introduce it as a constraint in our problem.

\section{Optimal TARGET COVERAGE WITH CONNECTIVITY CONSTRAINTS BETWEEN UAVS}

Let $\mathcal{U}$ be the set of available UAVs, and $\mathcal{N}$ be the set of targets that has to be monitored. Each target $n \in \mathcal{N}$ is assumed to be fixed, located at position $\left(x_{n}, y_{n}\right)$ on the 2D plane.

Each UAV $u \in \mathcal{U}$ can be located in the three dimensional space. Let $p=\left(x_{u}, y_{u}, h_{u}\right)$ be respectively the position $\left(x_{u}, y_{u}\right)$ of UAV $u$ in the 2D plane, and $h_{u}$ its altitude. We derive the observation radius $r_{u}^{h}$ of UAV $u$ in function of its altitude $h_{u}$ and its directional antenna half beamwidth $\theta$. The coverage area of UAV $u$ on the 2D plane, given the visibility angle $\theta$ and the altitude $h_{u}$, is represented by a disk of radius bounded by:

$$
r_{u}^{h} \leq h_{u} \cdot \tan \left(\frac{\theta}{2}\right)
$$

The higher the altitude, the longer the radius. However deploying UAVs at high altitude degrades the wireless signal. We thus seek at minimizing the altitude of the deployed UAVs in order to maximize the coverage quality.

We say that an UAV $u \in \mathcal{U}$ covers target $n \in \mathcal{N}$ if the distance between its projection on the 2D plane ant the target $d_{u n}=\sqrt{\left(x_{u}-x_{n}\right)^{2}+\left(y_{u}-y_{n}\right)^{2}}$ is below the observation radius $r_{u}^{h}$ of the UAV. Similarly, for airto-air communications, an UAV $u$ can communicate with another UAV $v$ if their distance on the $3 D$ plane $D_{u v}=$ $\sqrt{\left(x_{u}-x_{v}\right)^{2}+\left(y_{u}-y_{v}\right)^{2}+\left(h_{u}-h_{v}\right)^{2}} \leq R_{u}$, where $R_{u}$ is the communication range of UAV $u$.

For efficient data collection, we enforce the deployed UAVs to be connected with each other and with a fixed base station $b$ located on the ground at coordinates $\left(X_{b}, Y_{b}, 0\right)$.

\section{A. Problem definition}

The goal is to deploy UAVs and choose their respective position and altitude in the set of possible positions $P$ so that:

- all the mobile targets are covered by at least one UAV ;

- all the UAVs are connected with each other and to the base station to efficiently collect information.

Placing an UAV to monitor the targets involves cost considerations. We fix a given $\operatorname{cost} c_{u}$ associated with the deployment of UAV $u$ to a given position. This cost $c_{u}$ can be related to energy consideration, monitoring costs, etc. And if $c_{u}$ is fixed and equal to 1 for all the UAVs, our problem seek at minimizing the number of deployed UAVs.

The objective of our problem is to minimize the total cost of the UAVs used to cover all the targets, and their altitude. Given the definition of the coverage radius of the UAVs presented in the previous section, one can see that changing the UAV altitude impacts the coverage and the line-of-sight links to ground users. On the one hand, the coverage area grows with altitude, so the ground targets can be more covered with one UAV. On the other hand, the wireless communications with the targets degrade with altitude as path loss increases, so the coverage is not satisfying.

\section{B. Linear model for the target coverage problem}

In this section, we first present a linear programming formulation to deal with the problem of covering the ground targets with a set of connected UAVs, minimizing jointly the cost of the deployment and the maximum altitude of the UAVs.

Let $z_{p}^{u}$ be a binary variable indicating if UAV $u$ is deployed at position $p$. And let $\chi_{n}^{u}$ be another binary variable stating if UAV $u$ covers target $n$. 
The linear program is thus the following:

$$
\begin{array}{r}
\min \left(\sum_{p \in P} \sum_{u \in \mathcal{U}} c_{u} \cdot z_{p}^{u}+\max _{u \in U, p \in P} h_{u}(p) \cdot z_{p}^{u}\right) \\
\sum_{p \in P} z_{p}^{u} \leq 1, \forall u \in \mathcal{U} \\
\sum_{u \in \mathcal{U}} \chi_{n}^{u} \geq 1, \forall n \in \mathcal{N} \\
\chi_{n}^{u} \leq \sum_{p \in P} z_{p}^{u} \cdot \frac{r_{u}^{h}}{d_{u n}}, \forall u \in \mathcal{U}, n \in \mathcal{N} \\
z_{p}^{u}, \chi_{n}^{u} \in\{0,1\}
\end{array}
$$

The objective function (1) jointly minimizes the cost of the UAV deployment, and their maximum altitude $h_{u}$. Minimizing the maximum assigned altitude ensures a better coverage for all the ground targets in comparison to minimizing the sum of the altitudes. Indeed, it is better to have more UAVs at a low altitude to cover the targets and ensure a good coverage, than having some UAVs at an high altitude with a degraded communication quality.

Constraints (2) state that an UAV can be placed at most at one location. Constraints (3) verify that all the targets are covered by at least one UAV. And constraints (4) ensure that a target cannot be covered by an UAV that is not deployed or at distance greater than the transmission radius of the UAV.

\section{Ensuring connectivity among the UAVs}

We want to ensure connectivity among the UAVs in order to collect efficiently information from the ground. We assume that there is a base station $b$ collecting information from position $\left(X_{b}, Y_{b}, 0\right)$.

In order to model the connectivity constraints, we use inequalities related to the existence of a flow in the graph induced by the UAVs. To do so, we introduce variables $f_{u v} \in \mathbb{R}$ modeling the amount of flow sent between UAVs $u$ and $v$. The goal is to ensure the existence of a flow between the base station and all the deployed UAVs.

Connectivity constraints for our problem are thus:

$$
\begin{gathered}
\sum_{v \in \mathcal{U}, v \neq u} f_{u v}-\sum_{v \in \mathcal{U}, v \neq u} f_{v u} \\
=\left\{\begin{array}{cc}
\sum_{v \in \mathcal{U}} \sum_{p} z_{p}^{v} & \text { if } u=b \\
-\sum_{p} z_{p}^{u} & \text { if } u \neq b
\end{array}, \forall u \in \mathcal{U}\right. \\
f_{u v} \leq \sum_{p} z_{p}^{u} \cdot \frac{R_{u}}{D_{u v}} \cdot|\mathcal{U}|, \quad \forall u, v \in \mathcal{U} \\
f_{u v} \leq \sum_{p} z_{p}^{v} \cdot \frac{R_{u}}{D_{u v}} \cdot|\mathcal{U}|, \quad \forall u, v \in \mathcal{U} \\
f_{u v} \in \mathbb{R}
\end{gathered}
$$

Since we don't know exactly how many UAVs are deployed to cover the targets, the amount of flow sent by the base station to communicate with the UAVs is equal to $\sum_{u \in \mathcal{U}} \sum_{p} z_{p}^{u}$ (Constraints (6)) corresponding to the number of deployed UAVs in the solution. If UAV $u$ is not deployed, then all its associated $z$ variables are forced to be equal to 0 , which ensure no flow's passing through node $u$. Otherwise, $u$ is deployed, receive one unit of flow (because $\sum_{p} z_{p}^{u}=1$ ) and forward the rest of the flow. Finally, flow can be sent between two UAVs if they are within communication range of each other (Constraints (7) and (8)). This means that UAV $u$ is connected with UAV $v$ if the communication range of $u$ is greater than its distance from $v$.

\section{Multi-objective linear model}

From previous model, we can analyze more deeply the trade-off between two antagonistic objectives: minimizing deployment cost and UAV altitude. Indeed, the higher the altitude, the larger the coverage. And so we need less drones to cover all the targets. But we also want to minimize the altitude in order to ensure good wireless communications quality. So limiting the altitude inextricably increases the number of deployed UAVs. The two parts of objective function (1) can actually be optimized separately in an effective multi-objective linear program.

In the following, we present our multi-objective linear program for an optimal target coverage with connectivity constraints among the UAVs, optimizing two separate objective functions:

$$
\begin{array}{r}
\min \sum_{p \in P} \sum_{u \in \mathcal{U}} c_{u} \cdot z_{p}^{u} \\
\min \max _{u \in U, p \in P} h_{u}(p) \cdot z_{p}^{u} \\
\text { s.t. }(2)-(9)
\end{array}
$$

Since these two objectives are in conflict, it is not relevant to combine them in an effective optimization problem. Indeed, pursuing the optimization of the UAVs altitude inextricably increases the number of needed UAVs to cover all the targets. And limiting the cost of deployment implies trying to use the least number of UAVs, which increases their altitude in order to get larger coverage areas.

Consequently, for such a multi-objective optimization problem in which the objectives cannot be optimized simultaneously, the concept of Pareto optimality was introduced into the evaluation system. The main idea to study trade-offs between the two metrics (10) and (11) is to find out all the possible nondominated solutions of the optimization problem. If a solution is non-dominated within the whole solution space, it is not possible to improve one of the metrics without worsening at least one of the other metrics. Each multi-objective problem has a set of Pareto-optimal solutions defined as the set of nondominated solutions. The set of all non-dominated solutions is the Pareto front. The Pareto front provides a set of solutions that can be chosen depending on the application requirements.

More precisely, each non-dominated solution represents a different optimal trade-off between the objectives. In order to generate Pareto-optimal solutions of our target covering problem with connectivity, we use the $\epsilon$-constraint method that transforms the bi-objective problem into a sequence of parametrized single objective problems such that the optimum 
of each single-objective problem corresponds to a Paretooptimal solution [11], [12]. We thus generate and solve monoobjective optimization problems of the form:

$$
\left\{\begin{array}{l}
\min f^{i}(x) \\
\text { s.t. } f^{j}(x) \leqslant \epsilon^{j}, \forall j \neq i
\end{array}\right.
$$

The $\epsilon^{i}$ are chosen such that $\overline{f^{i}} \leqslant \epsilon^{i}$, where $\overline{f^{i}}$ corresponds to the optimum value of the mono-objective problem minimizing only objective $f^{i}$.

\section{RESUlts}

The model presented in the previous section has been implemented in Java language and solved using IBM Cplex solver 12.7.1. It has been solved on an Intel(R) Core(TM) i7-5500U CPU, $2.40 \mathrm{GHz}, 16 \mathrm{~Gb}$ RAM machine, under Microsoft 8.1 Professional operation system.

In the following, we present results for the optimal target coverage, with connectivity constraints among the UAVs. We show the effectiveness of our linear model by presenting optimal results and their computational time for various topologies. Moreover, we study the trade-off between deployment cost and altitude of the UAVs by solving the bi-objective linear model with the $\epsilon$-constraint method to obtain optimal solutions on the Pareto front. We choose to define the fair optimal solution of the problem and analyze its efficiency. Finally, we compare our solutions with the one obtained in an optimal target coverage without connectivity constraints, to highlight the cost of connecting the UAVs.

\section{A. Scenario description}

Instances are deployed in a square area of size $100 \mathrm{~m} \times$ $100 \mathrm{~m}$. We choose randomly the $2 \mathrm{D}$ coordinates of the targets on this area. For each random set of targets computed, we divide the area considered into equal squares in which one possible point is located in the center of the square. In this way, the candidate sites for placing a flying drone form a regular grid. For each point of coordinate $\left(x_{p}, y_{p}\right)$, we set the allowed altitudes to $\{10 \mathrm{~m}, 25 \mathrm{~m}, 45 \mathrm{~m}\}$. The base station is placed at coordinates $(0.0,0.0,0.0)$. We then generated instances of size between 5 and 50 targets, and between 50 and 300 possible points for the UAVs. We assume that we have an infinite number of available UAVs so that the model allows to deploy as many UAVs as necessary to fulfill the constraints of our problem. The associated deployment cost for each UAV is set to 1 . The visibility angle of the UAVs is set to 60 degrees, and their communication range to $30 \mathrm{~m}$. For each size of $\mathcal{N}$ and $P$, we compute 5 different random topologies. We summarize results with the mean value for each topology size.

\section{B. Trade-off analysis between deployment cost and altitude}

In this section, we present results obtained by solving our biobjective linear program with the $\epsilon$-constraint method in order to get optimal solutions on the Pareto front. To demonstrate the utility of this approach, we generate sets of non-dominated solutions by iteratively solving $\epsilon$-constraint mono-objective optimization problems.
We first solve twice our model for each scenario described in the previous section, with one objective each. We thus obtain $\overline{f^{1}}$ the minimum deployment cost without any constraint about the altitude, and $\overline{f^{2}}$ the smallest altitude for which we can obtain a connected set of UAVs covering the targets, without any constraint on the set's cost. Then, we iteratively solve the mono-objective problem minimizing (11) with the $\epsilon$ constraint $\sum_{p \in P} \sum_{u \in \mathcal{U}} c_{u} \cdot z_{p}^{u} \leq \epsilon, \epsilon$ varying from the deployment cost obtained for $\overline{f^{1}}$ to the one for $\overline{f^{2}}$.

1) Analysis of Pareto fronts: We depict in Figure 1 results obtained when the altitude varies between $\overline{f^{1}}$ and $\overline{f^{2}}$. Indeed, when the highest altitude is already reached when minimizing only it, then the optimal solution is the one with smallest cost, i.e. $\overline{f^{1}}$ is the best solution. In our scenarios, it usually happens when $\min \max _{u \in U, p \in P} h_{u}(p) \cdot z_{p}^{u}=45$ the maximum possible altitude. It means that there is no connected set of UAVs, covering the targets, with all the UAVs at altitude below $45 \mathrm{~m}$. But it can also happen that the altitude is equal for $\overline{f^{1}}$ and $\overline{f^{2}}$. For example, it happens with 5 targets and 147 possible points that the optimal coverage is guaranteed with all UAVs at altitude $10 \mathrm{~m}$. In such cases, the optimal solution $\overline{f^{1}}$ minimizing the cost (10) is optimal in terms of cost and altitude.

In main cases, it is possible to decrease the maximum altitude by adding additional UAVs as depicted in the Pareto fronts (Figure 1). Since the objective function (11) minimizes the maximum altitude, we observe that Pareto fronts quickly decrease to $\overline{f^{2}}$. Indeed, possible values taken by objective (11) depends on the scenario considered (i.e. 3 possibilities in our case). For our given scenario that corresponds to practical cases, it is thus possible to attain the minimum altitude with a low number of deployed UAVs. Figures 1 show that the minimum altitude is attained with at most 6 additional UAVs, compared to the optimum of the first objective function $\overline{f^{1}}$. For topologies with a low number of ground targets (i.e. 10 and 15 targets), the maximum is 3 . This is a good compromise between deployment cost and communication quality as expected for our problem.

One last example arises for 10 targets and 300 possible locations for the UAVs. $\overline{f^{1}}$ gives an altitude of $45 \mathrm{~m}$ for 6 connected UAV s covering the targets. On the contrary, $\overline{f^{2}}$ gives a solution of 20 connected UAVs at altitude $10 \mathrm{~m}$. Therefore, the Pareto front goes from $45 \mathrm{~m}$ to $25 \mathrm{~m}$, and then to $10 \mathrm{~m}$ (left upper figure). In this case, it is interesting to see that 6 additional UAVs are necessary to obtain the best attainable altitude. This stays into the limit found for larger topologies.

From the observation of the Pareto fronts, we derive a definition of the best trade-off solution of our problem.

Definition 1. The fair optimal solution of the target covering problem with connectivity constraints among the UAVs corresponds to the solution $\left(c^{*}, h_{\max }^{*}\right)$ such that :

- $h_{\text {max }}^{*}=\overline{f^{2}}$ is the optimal solution of objective (11);

- $c^{*}$ is the minimum deployment cost obtained when attaining $\overline{f^{2}}$. 

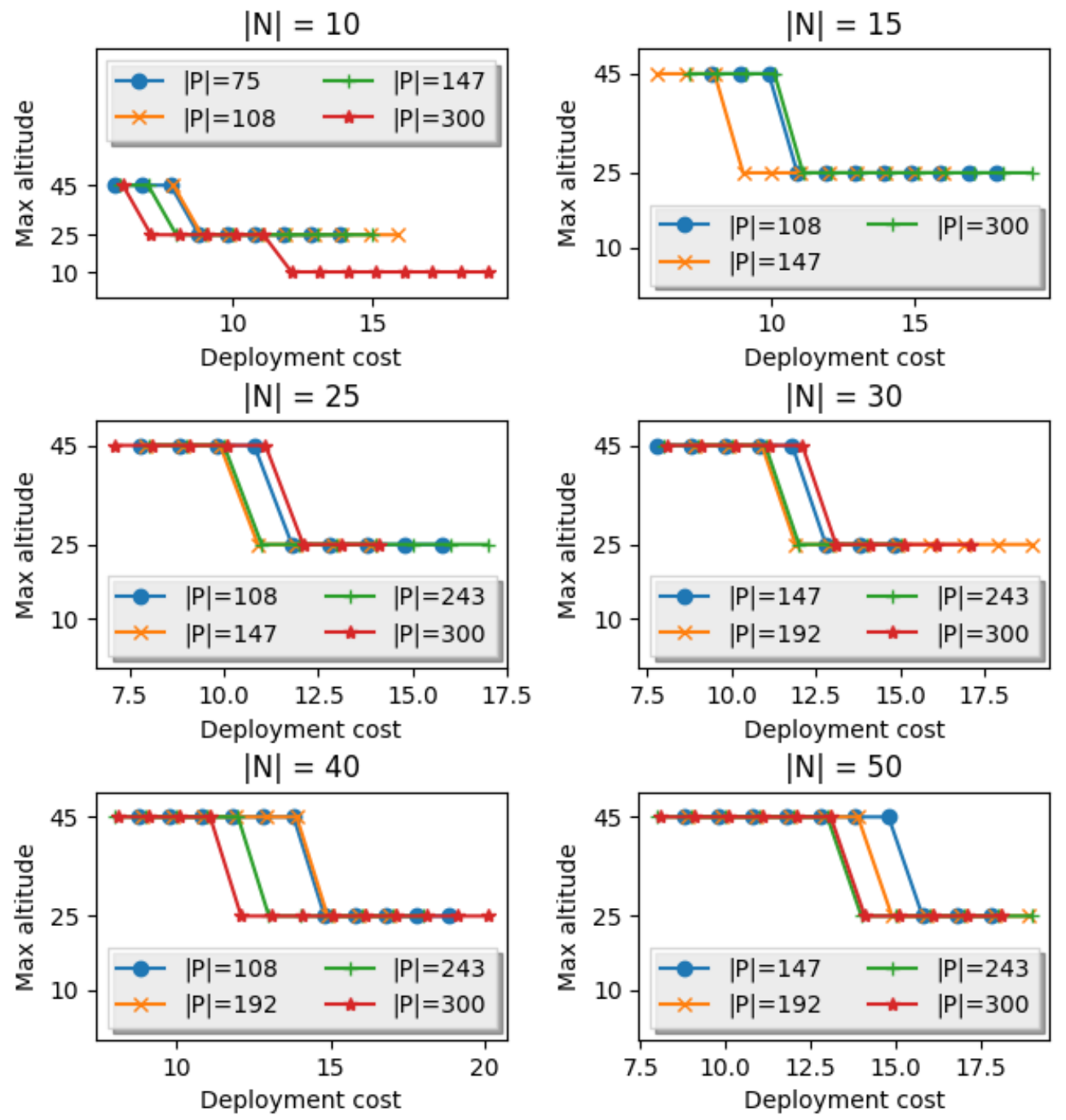

Fig. 1: Pareto fronts for different sizes of $\mathcal{N}$ and $P$.

This fair optimal solution allows us to propose one optimal solution in terms of altitude with minimum associated deployment cost. From this global observation, we derive a deeper analysis to validate the effectiveness of the fair solution. In the following, we show the efficiency of our solution by comparing it to other solutions of the Pareto front.

2) Validation of the fair optimal solution: We first claim that the fair optimal solution is efficient in terms of altitude. If we look at the sum of all the altitudes of the fair solution, compared to the solutions $\overline{f^{1}}$ and $\overline{f^{2}}$, we see that the fair optimal solution provides an UAV backbone at lower altitude (see Figure 2). Indeed, $\overline{f^{1}}$ (named as opt cost in the figure) does not optimize altitude at all, but seek to deploy the smallest number of UAVs (at high altitude). Solution $\overline{f^{2}}$ (called opt altitude in the figure) optimizes the altitude, but gives a solution with more UAVs, leading to a larger value of the altitude's sum. Therefore the fair optimal solution provides a better solution since the sum of the altitudes is lower than the two mono-objective solutions.

Another metric validating the choice of the fair optimal solution is the coverage density of the solution. It corresponds to the mean number of covered targets by the deployed UAVs. In a solution, we can deploy UAVs only to forward traffic to the base station, thus covering no target. On the contrary, if one UAV covers too many targets, then it leads to bad air-to-ground communications due to wireless interferences. We analyze the coverage density in Figure 3 in which we represent, for a given number of targets, a maximum of three bars representing the coverage density for respectively 108,147 , and 300 possible points. We remark that the coverage density of the fair optimal solution has value between the density of $\overline{f^{2}}$ and $\overline{f^{1}}$. It cannot be lower than $\overline{f^{2}}$ because this solution deploys more UAVs 

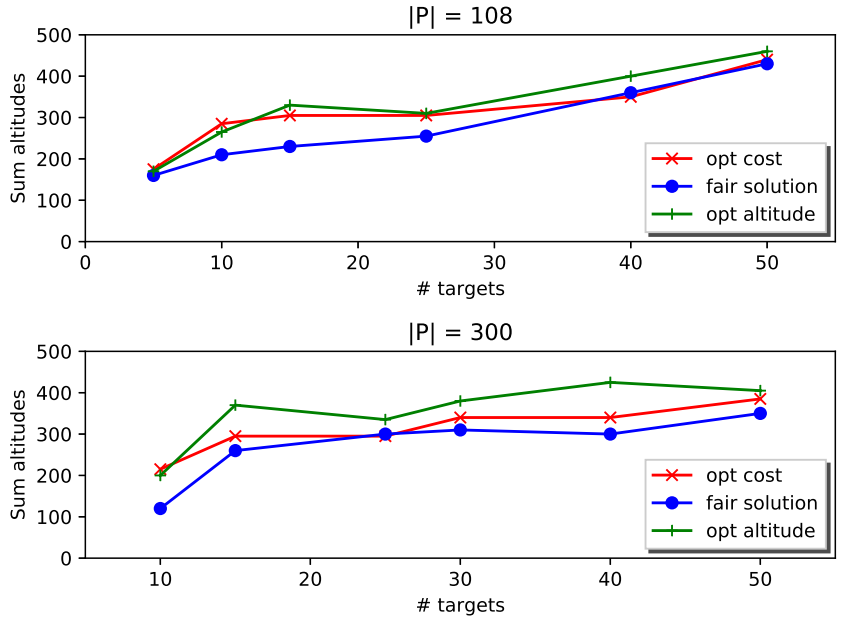

Fig. 2: Sum of altitudes of deployed UAVs

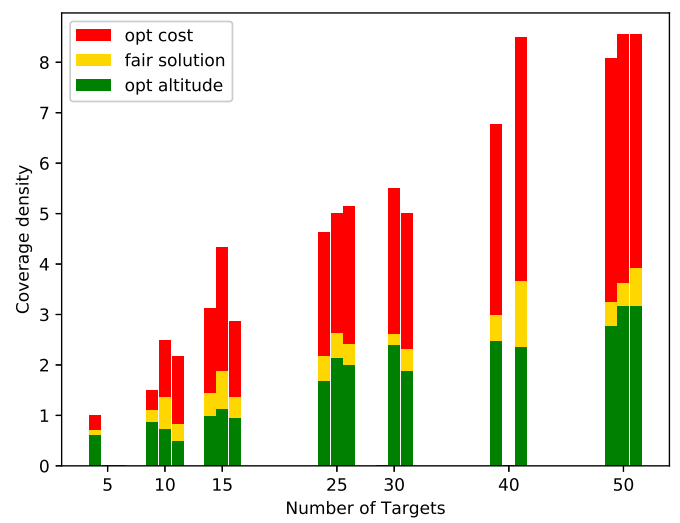

Fig. 3: Mean number of targets covered by the deployed UAVs for $|P|=108,147,300$.

at low altitude, thus balancing the target's coverage. On the contrary, $\overline{f^{1}}$ minimizes the number of deployed UAVs. They are placed at high altitude, covering more targets per UAV. But the fair optimal solution has a low coverage density, usually closed to the one of $\overline{f^{2}}$. This is a good result since it increases the air-to-ground communication quality while ensuring the lowest altitude for the deployed UAVs.

\section{Model efficiency}

In Table I, we show detailed results of the different scenarios studied. We present the deployment cost, the altitude, and the computational time obtained for the fair optimal solution defined in the previous section and the solution of the non connected case, for each size of $\mathcal{N}$ and $P$.

1) Computational time: We can first highlight the fact that our model always find an optimal solution of the target covering problem with connectivity constraints among the UAVs in reasonable time. Even for more than 200 possible points, inducing more than 10000 possible links for the connected
TABLE I: Computational results of our deployment model.

\begin{tabular}{|c|c|c|c|c|c|c|c|}
\hline \multicolumn{3}{|c|}{ Topology } & \multicolumn{2}{|c|}{$\begin{array}{c}\text { Optimal solution } \\
\text { without connectivity }\end{array}$} & \multicolumn{3}{|c|}{$\begin{array}{l}\text { Fair optimal solution } \\
\text { with connectivity }\end{array}$} \\
\hline$|\mathcal{N}|$ & $|P|$ & $|E|$ & $|\mathcal{U}|$ & $\max h_{u}$ & $|\mathcal{U}|$ & $\max h_{u}$ & Time (s) \\
\hline 5 & 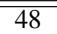 & 510 & 3 & $\overline{45}$ & $\overline{c 6}$ & 45 & $\begin{array}{l}0.453 \\
\end{array}$ \\
\hline 10 & - & - & 5 & 45 & 8 & 45 & 0.14 \\
\hline 15 & - & - & 5 & 45 & 8 & 45 & 0.203 \\
\hline 20 & - & - & 6 & 45 & 9 & 45 & 0.266 \\
\hline 25 & - & - & 7 & 45 & 10 & 45 & 0.265 \\
\hline 30 & - & - & 6 & 45 & 10 & 45 & 0.266 \\
\hline 40 & - & - & 5 & 45 & 8 & 45 & 0.188 \\
\hline 50 & - & - & 7 & 45 & 9 & 45 & 0.391 \\
\hline 5 & 75 & 982 & 2 & 45 & 6 & 45 & 0.421 \\
\hline 10 & - & - & 7 & 25 & 9 & 25 & 0.281 \\
\hline 15 & - & - & 4 & 45 & 10 & 45 & 4.375 \\
\hline 20 & - & - & 5 & 45 & 10 & 45 & 2.032 \\
\hline 25 & - & - & 5 & 45 & 10 & 45 & 10.422 \\
\hline 30 & - & - & 7 & 45 & 12 & 45 & 16.562 \\
\hline 40 & - & - & 6 & 45 & 11 & 45 & 3.906 \\
\hline 50 & - & - & 7 & 45 & 12 & 45 & 14.844 \\
\hline 5 & 108 & 1974 & 3 & 25 & 7 & 25 & 1.016 \\
\hline 10 & - & - & 7 & 25 & 9 & 25 & 1.891 \\
\hline 15 & - & - & 9 & 25 & 11 & 25 & 7.656 \\
\hline 20 & - & - & 4 & 45 & 8 & 45 & 7.422 \\
\hline 25 & - & - & 10 & 25 & 12 & 25 & 1.328 \\
\hline 30 & - & - & 6 & 45 & 9 & 45 & 3.985 \\
\hline 40 & - & - & 14 & 25 & 15 & 25 & 0.5 \\
\hline 50 & - & - & 18 & 25 & 19 & 25 & 0.39 \\
\hline 5 & 147 & 3806 & 4 & 10 & 5 & 10 & 1.359 \\
\hline 10 & - & - & 7 & 25 & 8 & 25 & 0.875 \\
\hline 15 & - & - & 8 & 25 & 9 & 25 & 1.562 \\
\hline 20 & - & - & 5 & 45 & 8 & 45 & 1.359 \\
\hline 25 & - & - & 10 & 25 & 11 & 25 & 2.719 \\
\hline 30 & - & - & 11 & 25 & 13 & 25 & 1.656 \\
\hline 40 & - & - & 6 & 45 & 9 & 45 & 45.609 \\
\hline 50 & - & - & 15 & 25 & 16 & 25 & 2.578 \\
\hline 5 & 192 & 6228 & 3 & 25 & 9 & 25 & 274.735 \\
\hline 10 & - & - & 7 & 25 & 9 & 25 & 181.062 \\
\hline 15 & - & - & 10 & 25 & 12 & 25 & 658.766 \\
\hline 20 & - & - & 11 & 25 & 12 & 25 & 270.125 \\
\hline 25 & - & - & 5 & 45 & 8 & 45 & 334.875 \\
\hline 30 & - & - & 11 & 25 & 12 & 25 & 218.515 \\
\hline 40 & - & - & 13 & 25 & 15 & 25 & 93.546 \\
\hline 50 & - & - & 15 & 25 & 15 & 25 & 3.468 \\
\hline 5 & 243 & 10278 & 4 & 25 & 5 & 25 & 2.531 \\
\hline 10 & - & - & 6 & 25 & 8 & 25 & 269.843 \\
\hline 15 & - & - & 9 & 25 & 10 & 25 & 6.312 \\
\hline 20 & - & - & 9 & 25 & 9 & 25 & 283.562 \\
\hline 25 & - & - & 9 & 25 & 11 & 25 & 1627.265 \\
\hline 30 & - & - & 10 & 25 & 12 & 25 & 680.828 \\
\hline 40 & . & - & 12 & 25 & 13 & 25 & 894.562 \\
\hline 50 & - & - & 13 & 25 & 14 & 25 & 704.859 \\
\hline 5 & 300 & 15102 & 4 & 25 & 6 & 25 & 5810.016 \\
\hline 10 & - & - & 10 & 10 & 12 & 10 & 2194.968 \\
\hline 15 & - & - & 9 & 25 & 11 & 25 & 4851.406 \\
\hline 20 & - & - & 10 & 25 & 10 & 25 & 621.593 \\
\hline 25 & - & - & 12 & 25 & 12 & 25 & 285.937 \\
\hline 30 & - & - & 12 & 25 & 13 & 25 & 3214.922 \\
\hline 40 & - & - & 12 & 25 & 12 & 25 & 5426.11 \\
\hline 50 & - & - & 14 & 25 & 14 & 25 & 70.5 \\
\hline
\end{tabular}

backbone, our model usually finds an optimal solution in less than 1000 seconds. This allows to optimally deploy UAVs for practical problems of enhancing the coverage and rate performances of wireless networks in emergency situations.

Moreover, we remark that the resolution time does not depend on the number of targets, but on the number of edges connecting the possible locations for the UAVs. So even for larger number of possible UAV locations, if the communication range between them is not too important, then the number of edges can stay bounded and the resolution time 


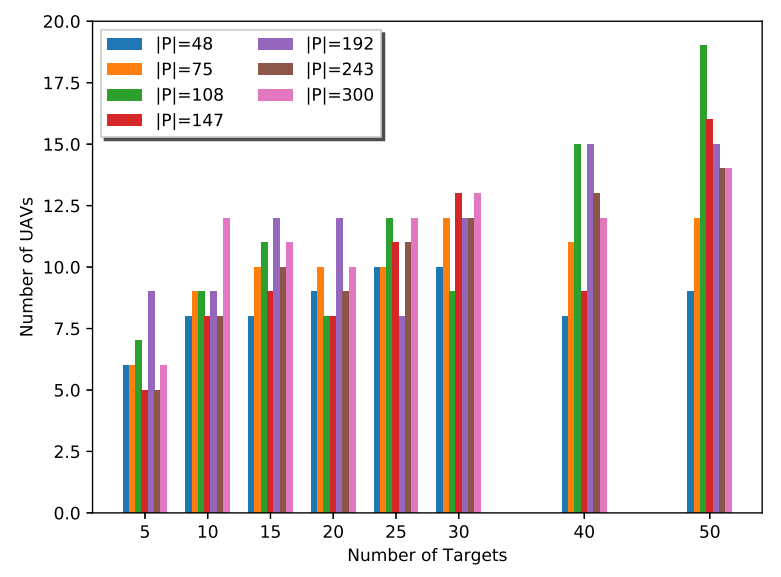

Fig. 4: Deployment cost of the optimal solutions.

low. This means that our model fits larger areas to monitor, and more possible altitudes for the UAVs. This is a promising result for future work.

2) Deployment cost: We can see on Figure 4 that the deployment cost increases linearly with the number of ground targets. The increase is even almost constant when the number of possible locations for the UAVs is small (i.e. 48 and 75) and the number of targets important (i.e. greater than 25). In such cases, covering the ground targets implies choosing the highest available altitude for the UAVs. Therefore, the coverage area is important and stays valid when the number of targets increases.

When the choice of locations for the UAVs is more important, then it becomes possible to cover the targets with a lower altitude. We obtain fair optimal solutions with a smaller maximum altitude, which implies having more flying drones, but ensures a better wireless communication quality.

Similarly, when the maximum altitude of the UAVs increases in a solution, we observe that the deployment cost decreases as the coverage area of the deployed UAVs is more important. It is illustrated in Figure 4 for $|P|=108$, the fair optimal solution when $|\mathcal{N}|=20$ or 30 has a lower deployment cost. It corresponds to solutions when the maximum altitude is $45 \mathrm{~m}$ instead of $25 \mathrm{~m}$ for the other values of $|\mathcal{N}|$. A solution with higher altitude is possible only if there is no connected set of UAVs for the lower possible altitudes, in particular when a target is located far from possible drone locations.

3) Cost of UAVs connectivity: Finally, we compare the optimal target coverage with and without the connectivity constraints among the deployed UAVs. This additional set of constraints enforces to use at least as many UAVs as in the optimal coverage without connectivity. Indeed, either the optimal solution for target coverage is a connected set of UAVs and with the base station, so it is an optimal solution of our problem. Or the optimal solution of the target coverage is not connected and we need additional UAVs to induce connectivity. We thus define the cost of connectivity, the additional amount (in terms of deployment cost) to pay to

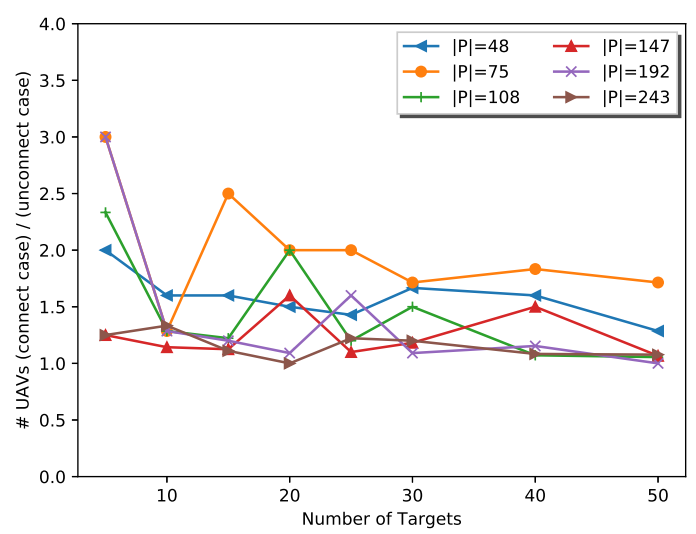

Fig. 6: Cost of UAVs connectivity.

get a connected set of UAVs, also connected with the base station.

Results of the unconnected case are presented in columns 4 and 5 of Table I. They first show that ensuring connectivity among the UAVs does not increase the maximum altitude of the flying devices. It is always possible to find a connected set of covering drones with the same maximum altitude as for the unconnected version. We start from the optimal solution of the covering problem without connectivity, and we only add forwarding drones at the same maximum altitude to connect the backbone network. A stronger result is to reduce the maximum altitude in the connected case. Let, for example, have an optimal solution with one drone at high altitude covering all the targets. This drone is not connected with the base station. Then, deploying several UAVs at a lower altitude, ensuring a complete coverage of the targets and the existence of a path until the base station is sometimes possible. If this case where the maximum altitude decreases in the connected case does not happen in our tests is because it needs various possible points and altitude. The number of needed UAVs to cover all the target at a low altitude can be important and not practically feasible.

Another result to point out concerns the cost of connectivity. As presented for one topology of 5 targets and 48 possible points for the UAVs in Figure 5, when the number of targets is low, then the number of additional UAVs needed in the connected case is important (maximum 3 times more for $|\mathcal{N}|=5$ and $|P|=243$ ). Indeed, depending on the position of the targets, it is necessary at least to connect with the base station located in the left bottom corner of the considered region. Some UAVs located closed to the base station are required to forward traffic collected from the targets.

On the contrary, when the number of targets increases, then the cost of connectivity decreases, as the covering constraints enforces to deploy more UAVs and so flying drones do not have to only forward traffic anymore. This remark is also pointed out on Figure 6 in which we present the evolution of the cost of connectivity in function of the number of targets 


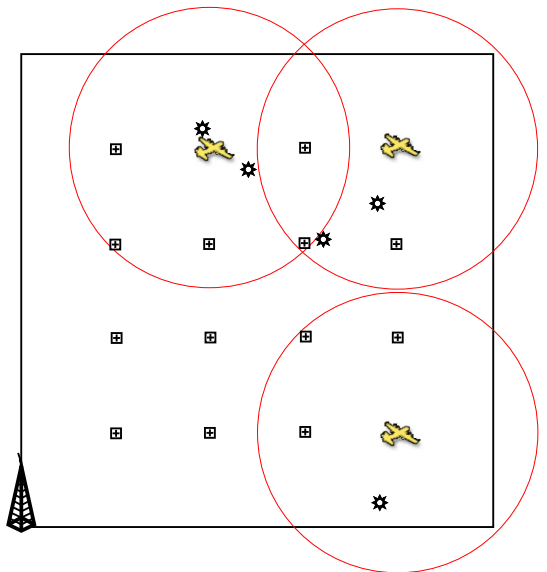

(a) Non connected optimal solution. 3 UAVs placed at max altitude 45 .

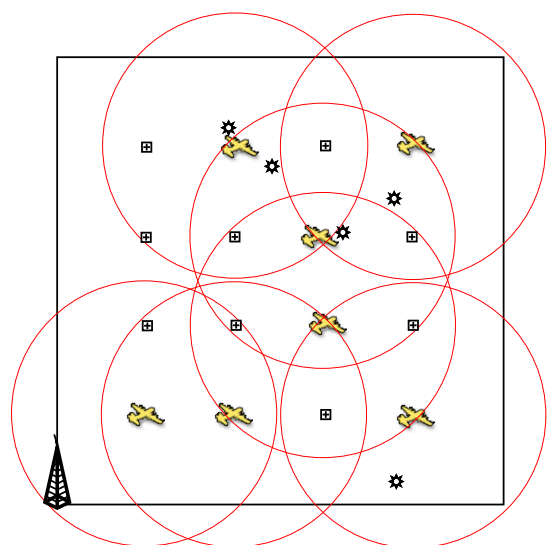

(b) Connected optimal solution. 7 UAVs placed at max altitude 45 .

Fig. 5: Optimal solutions obtained for 5 targets and 48 possible points (16 different 2D coordinates and 3 possible altitudes).

for different numbers of possible points. When the number of targets or possible points for the UAVs is important, then the cost of connectivity decreases to 1 , which is an advantage as monitoring areas are growing these days.

\section{CONCLUSION}

In this work, we address the problem of covering ground targets with Unmanned Aerial Vehicles monitoring the targets and forming a backbone connected network to collect efficiently information from the ground. We present an optimal bi-objective linear program to model the problem and obtain optimal solutions on the Pareto front in reasonable time for real size instances.

A study of the Pareto front helps analyzing the trade-off between deployment cost and altitude. Indeed, minimizing the altitude ensure good wireless communication quality for the data collection. We observe that our bi-objective model helps us provide a fair optimal solution minimizing the maximum altitude with a few number of additional UAVs than the optimum deployment cost solution. This solution thus balance efficiently deployment cost and communication quality.

A comparison with the usual problem of target covering without connectivity constraints has been performed and present the cost to pay in terms of additional UAVs deployed to ensure a connected backbone. Results show that this cost is low (and even null) for large scenarios in terms of number of targets and possible points.

This work constitutes a starting point to the study of the UAV deployment problem. Future work is about extending this model to deal with target mobility. Time is discretized and at each time slot we want to compute an optimal coverage with a connected set of UAVs. This extension will make the problem size explode. Sophisticated techniques like column generation, and branch and price, will thus be investigated in order to find effective solutions.

\section{REFERENCES}

[1] D. Zorbas, L. Di Puglia Pugliese, T. Razafindralambo, and F. Guerriero, "Optimal drone placement and cost-efficient target coverage," Journal of Network and Computer Applications, vol. 75, pp. 16 - 31, 2016.

[2] M. Mozaffari, W. Saad, M. Bennis, and M. Debbah, "Efficient deployment of multiple unmanned aerial vehicles for optimal wireless coverage," IEEE Communications Letters, vol. 20, no. 8, pp. 1647-1650, Aug 2016.

[3] L. Navarro-Serment, J. Dolan, and P. Khosla, "Optimal sensor placement for cooperative distributed vision," in Robotics and Automation, 2004. Proceedings. ICRA '04. 2004 IEEE International Conference on, vol. 1, April 2004, pp. 939-944 Vol.1.

[4] S. MartíNez and F. Bullo, "Optimal sensor placement and motion coordination for target tracking," Automatica, vol. 42, no. 4, pp. 661668, 2006.

[5] K. Dasgupta, M. Kukreja, and K. Kalpakis, "Topology-aware placement and role assignment for energy-efficient information gathering in sensor networks," in Proceedings. Eighth IEEE International Symposium on Computers and Communication. IEEE, 2003, pp. 341-348.

[6] Q. Wang, K. Xu, G. Takahara, and H. Hassanein, "Deployment for information oriented sensing coverage in wireless sensor networks," in Global Telecommunications Conference, (GLOBECOM). IEEE, 2006.

[7] K. Kar, S. Banerjee et al., "Node placement for connected coverage in sensor networks," in WiOpt'03: Modeling and Optimization in Mobile, Ad Hoc and Wireless Networks, 2003.

[8] F. Alduraibi, N. Lasla, and M. Younis, "Coverage-based node placement optimization in wireless sensor network with linear topology," in 2016 IEEE International Conference on Communications (ICC), May 2016.

[9] D. Zorbas, T. Razafindralambo, D. P. P. Luigi, and F. Guerriero, "Energy efficient mobile target tracking using flying drones," Procedia Computer Science, vol. 19, pp. $80-87,2013$, the 4th International Conference on Ambient Systems, Networks and Technologies (ANT 2013), the 3rd International Conference on Sustainable Energy Information Technology (SEIT-2013).

[10] L. D. P. Pugliese, F. Guerriero, D. Zorbas, and T. Razafindralambo, "Modelling the mobile target covering problem using flying drones," Optimization Letters, vol. 10, no. 5, pp. 1021-1052, 2016.

[11] M. Laumanns, L. Thiele, and E. Zitzler, "An adaptive scheme to generate the pareto front based on the epsilon-constraint method," in Practical Approaches to Multi-Objective Optimization, ser. Dagstuhl Seminar Proceedings, J. Branke, K. Deb, K. Miettinen, and R. E. Steuer, Eds., no. 04461, Dagstuhl, Germany, 2005.

[12] J.-F. Bérubé, M. Gendreau, and J.-Y. Potvin, "An exact -constraint method for bi-objective combinatorial optimization problems: Application to the traveling salesman problem with profits," European Journal of Operational Research, vol. 194, no. 1, pp. 39 - 50, 2009. 